DE DE GRUYTER OPEN

\section{Research Article}

(C) 2017 Beatrice Ugolini. This is an open access article licensed under the Creative Commons Attribution-NonCommercial-NoDerivs License (http://creativecommons.org/licenses/by-nc-nd/3.0/).

\title{
Insanity and Social Dangerousness: Philosophical Reflections on Italian Law
}

\author{
Beatrice Ugolini
}

\author{
Expert Judge of the Surveillance Court in Bologna (Italy)
} PhD in Law theories. Criminologist.

Doi: 10.1515/ajis-2017-0017

\begin{abstract}
According to Italian law 81/2014, social dangerousness derived only from individual personal characteristics, including mental health conditions. As a result, dangerousness is not perceived as related to a specific context, but on the contrary mainly caused by a psychic condition. Although forensic psychiatry and criminology have denied this axiom for a long time now, this ancient and traditional prejudice where a mentally ill person is dangerous as such, seems to have returned formally. Beyond the contingent issues that may have influenced Italian lawmakers in formulating this legislation, there is a question to ask: why does this union between mental illness/social dangerousness reappears in a cyclic and persistent way? Since imputability and social dangerousness are not topics studied exclusively by law and psychiatry, it is possible to express some philosophical considerations regarding the correlation between insanity and deviance and the ancient bond between insanity and evil. In assessing psychiatric social dangerousness, in order to reconcile instances of the rights of individual patients with the need to protect society, the law needs to take into account these hidden and intertwined issues.
\end{abstract}

Keywords: insanity, social dangerousness, deviance

\section{Introduzione}

II testo del D.L. 31 marzo 2014 n. 52, coordinato con la legge di conversione 30 maggio 2014, n. 81, recante "Disposizioni urgenti in materia di superamento degli ospedali psichiatrici giudiziari", all'art. 1 lett. b) stabilisce che la pericolosità sociale venga accertata "sulla base delle qualità soggettive della persona e senza tenere conto delle condizioni di cui all'articolo 133, secondo comma, numero 4, del codice penale. Allo stesso modo provvede il magistrato di sorveglianza quando interviene, ai sensi dell'articolo 679 del codice di procedura penale. Non costituisce elemento idoneo a supportare il giudizio di pericolosità sociale la sola mancanza di programmi terapeutici individuali». Nell'applicazione delle misure di sicurezza riservate ai soggetti con vizio totale o parziale di mente, il giudizio di pericolosità sociale potrà, dunque, essere desunto solo dalle qualità soggettive della persona. La pericolosità sarebbe, di conseguenza, decontestualizzata e determinata principalmente dalla patologia psichica. Pare, dunque, ripresentarsi, in veste giuridica, il tradizionale e secolare pregiudizio secondo cui il malato psichico è, di per sé, pericoloso. Al di là delle questioni contingenti che possono aver influenzato il legislatore italiano nella formulazione di

${ }^{1}$ II D.L. n. 52/2014 si inscrive nel progressivo e faticoso superamento degli Ospedali Psichiatrici Giudiziari (Opg), avviato in Italia dal 2008, sancito dal D.L. 211/2011, convertito poi nella legge 17 febbraio 2012 n. 9 , e rallentato da una serie di proroghe. 
tale normativa, una domanda che risulta interessante porsi è: perché si riaffaccia, in modo così ciclico e persistente, il connubio malattia mentale/pericolosità sociale?

\section{II dibattito sul ritorno della pericolosità sociale presunta}

In Italia, numerose e autorevoli sono state le critiche a quello che sembra il ritorno a una visione lombrosiana del reo malato psichico. Chi ha, in particolare, raccolto e dato voce a tali critiche è stato il Tribunale di Sorveglianza di Messina che, con Ordinanza n. 247 del $2014^{2}$, ha sollevato la questione di legittimità costituzionale, accusando, in sostanza, il legislatore «di aver riportato indietro le lancette dell'orologio, quando ancora era imperante il modello cartesiano classico della rigida separazione tra res cogitans (la psiche) e res extensa (il corpo), fondamento della oramai anacronistica psichiatria organicista» (Pugiotto, 2015, p. 3). Nell'Ordinanza, viene evidenziato come la pericolosità sociale decontestualizzata, considerata cioè in maniera isolata da quello che è l'ambiente di vita del soggetto, impedisce, di fatto, che il giudice possa svolgere in modo completo e circostanziato l'accertamento in concreto della reale pericolosità (o non pericolosità) sociale. Ciò comporta l'introduzione, in forma implicita e ingiustificata, di una costituzionalmente criticabile presunzione legislativa di pericolosità (o di non pericolosità), priva, in ogni caso, di una solida motivazione scientifica ${ }^{3}$. A parere del Tribunale, inoltre, viene adombrato un modello criminologico di genere unifattoriale, individualistico, fondato su cause esterne e ormai ritenuto, da molto tempo, scientificamente non più valido (Cfr. Bianchetti, 2014). Ciò che, in definitiva, viene denunciato è il pericolo di accrescere a dismisura, in sede di valutazione prognostica, il peso peculiare della malattia mentale, arrivando in tal modo a ristabilire l'ormai antiquato automatismo per cui il malato psichico è pericoloso in quanto tale (Massaro, 2015, p. 1360). Al riguardo, Pellissero (2014, pp. 922-924) parla di «nuova forma mascherata di pericolosità sociale presunta» e sottolinea come la pericolosità vada intesa in una «accezione situazionale, perché il soggetto non è una monade, ma va calato nel contesto sociale e familiare di riferimento». Nel diritto penale, la nozione di pericolosità sociale situazionale «è alla base delle pronunce della Corte costituzionale che nel 2003 e 2004 hanno imposto il principio di sussidiarietà dell'OPG rispetto alla libertà vigilata terapeutica, che va privilegiata come misura di controllo del rischio di recidiva».

La Corte costituzionale, con la sentenza n. 186 del 2015, ha dichiarato infondata la questione di illegittimità costituzionale posta dal Tribunale di Messina. Nella sentenza si chiarisce che il limite stabilito dalla D.L. 31 marzo 2014 n. 52 non è, in generale, relativo alla pericolosità sociale, ma ha l'obiettivo di riservare le misure estreme, notevolmente vincolanti per la libertà personale, alle sole situazioni in cui sono le condizioni psichiche della persona a renderle indispensabili. $\dot{E}$, dunque, una disposizione che va intesa in senso ampio, nell'ambito di una serie di provvedimenti legislativi che mirano alla chiusura degli ospedali psichiatrici giudiziari; ciò, tuttavia, per i soggetti infermi o seminfermi di mente, non ha mutato, neppure di riflesso, la nozione di pericolosità sociale, poiché l'intervento normativo riguarda i criteri di scelta tra le misure di sicurezza e le condizioni per l'applicazione delle misure detentive ${ }^{4}$. In altri termini, la contestata disposizione dell' art. 1 lett. b) rappresenterebbe, in realtà, una norma a tutela della libertà del cittadino e risponderebbe all'esigenza di evitare, da un lato, che le condizioni di svantaggio economico e deprivazione sociale diventino decisive nell'attribuzione della patente di "soggetto socialmente pericoloso" e, dall'altro, che l'internamento possa dipendere da disservizi organizzativi, come, ad esempio, l'impossibilità, per i Dipartimenti di salute mentale, di prendere in carico il soggetto. (Massaro, 2015).

Anche a seguito dell'indagine messa in atto, nella scorsa XVI Legislatura, dalla Commissione d'inchiesta del Senato sulla qualità del Servizio Sanitario Nazionale, il legislatore ha ritenuto opportuno «evitare che il soggetto paghi colpe non sue, ogni qual volta il suo internamento in Opg

2 Tribunale di sorveglianza di Messina, Ordinanza n. 247 (Atto di promovimento), 16 luglio 2014, in http://www.gazzettaufficiale.it/eli/id/2015/01/14/14C00357/s1. Sito visitato il 10/10/2014.

3 Tribunale di sorveglianza di Messina, Ordinanza n. 247, cit.

${ }^{4}$ Corte cost., sentenza 186/2015, in http://www.giurcost.org/decisioni/2015/0186s-15.html. Sito visitato il 09/10/2016. 
viene disposto o prorogato esclusivamente per un deficit organizzativo dei servizi di prossimità o per l'indisponibilità dei familiari a farsene carico». In sostanza, un malato indigente, che vive ai margini della società, privo di alloggio e trascurato dai Servizi non può divenire, per questi motivi, socialmente pericoloso e ritrovarsi, poi, in Opg. Cosa, peraltro, piuttosto frequente attraverso il ricorso alla cosiddetta pericolosità sociale latente: «nell'indisponibilità familiare o territoriale, il magistrato proroga la misura di sicurezza detentiva (nonostante ne sia scemato il presupposto soggettivo, come attestato dall'osservazione clinica dell'internato), non potendosi escludere che, extra moenia, potrebbe riacutizzarsi la sua pericolosità sociale» (Pugiotto, 2015, pp. 2-3).

La situazione sociale e finanziaria dell'individuo e l'assenza di un piano terapeutico non possono più, quindi, giustificare la pericolosità sociale e il conseguente internamento e non motivano più neppure le proroghe. Attualmente, invece, un malato, abbandonato a se stesso, privo di qualsiasi aiuto, anche se gli spetterebbe di necessità, può essere considerato socialmente pericoloso. Con la recente normativa, si vorrebbe, dunque, evitare che l'Opg diventi il luogo in cui rinchiudere chi risulta, di fatto, abbandonato dalla collettività e chi, in seguito alle proroghe delle misure di sicurezza, paga le colpe di una inadeguata o inadempiente presa in carico dei Servizi sociali e/o sanitari ${ }^{5}$. Nonostante le lodevoli intenzioni e la chiara legittimità riconosciuta dalla Corte costituzionale, il rischio più consistente della L. 81/2014 rimane, comunque, quello di un internamento disposto considerando prioritariamente l'infermità mentale del soggetto; risulta, inoltre, difficoltoso capire se il fine plausibile sia davvero quello del ritorno di una concezione basata sulla sola valutazione della "malattia", invece che del "malato" nella sua interezza, con la conseguente reintroduzione dell'automatismo "infermità mentale = internamento" (Massaro, 2015).

La fattispecie della pericolosità sociale presunta è stata abrogata nel 1986 dalla legge 663. In origine, il codice penale del 1930 prevedeva, infatti, due forme di pericolosità: quella accertata di volta in volta dal Giudice, secondo quanto stabilito dall'art. 133 c.p., e quella presunta ex art. 204 c.p., in base alla quale il sofferente psichico autore di reato veniva "automaticamente" ritenuto socialmente pericoloso e, pertanto, internato. Già con l'introduzione della riforma Basaglia, nel 1978 , si era sgretolata la finzione secondo cui veniva attribuita natura terapeutica a provvedimenti di carattere solo custodialistico. Alla fondamentale legge 180, tuttavia, non segui mai un coerente piano di revisione della disciplina, soprattutto in merito agli Opg: negli anni a seguire, la giurisprudenza cercò di intervenire con provvedimenti che potessero adeguare il trattamento del sofferente psichico alle novità introdotte da Basaglia, ma l'impostazione di fondo rimase, e rimane, sospesa tra istanze di difesa sociale e istanze terapeutiche. La sentenza della Corte Costituzionale n. 253/2003 ha, in particolare, sancito il superamento dell'automatismo tra pericolosità sociale per malattia mentale e applicazione della misura di sicurezza in OPG. Tale sentenza si è, infatti, pronunciata sulla necessità di adottare misure di sicurezza alternative, laddove previste, idonee ad assicurare, al contempo, cure adeguate e controllo sociale. La Corte Costituzionale ha dichiarato illegittimo l' articolo 222 c.p. nella parte in cui non consente al giudice, nei casi ivi previsti, di adottare una diversa misura di sicurezza idonea ad assicurare adeguate cure dell'infermo di mente e a far fronte alla sua pericolosità sociale. Così come per il minore, infatti, anche per l'infermo di mente l'automatismo di una misura segregante e "totale" come il ricovero in OPG, imposta pur quando appaia in concreto inadatta, infrange l'equilibrio costituzionalmente necessario e viola esigenze essenziali di protezione dei diritti della persona, in particolare il diritto alla salute di cui all'articolo 32 della Costituzione (Bandini \& Rocca, 2010, p. 105).

Sino alla L. 81/2014, comunque, pareva ormai definitivamente smantellata l'indissolubilità del binomio pericolosità sociale/infermità mentale: quest' ultima rappresenta, così, non più una causa particolare di pericolosità, ma un qualsiasi fattore che, interagendo con gli altri, può esercitare un'efficacia criminogena. In altri termini, la pericolosità sociale dell'infermo di mente non poteva più essere ritenuta automaticamente presente, ma deve essere accertata non soltanto sulla base di valutazioni di natura medico-psichiatrica, ma secondo i criteri di valutazione stabiliti dall' art. 133 c.p.; spetta, inoltre, al magistrato di sorveglianza procedere, in qualsiasi momento, a un riesame

\footnotetext{
${ }^{5}$ Cfr. http://www.stopopg.it/taxonomy/term/4. Sito visitato il 05/01/2016.
} 
della pericolosità (Bandini \& Rocca, pp. 106-108).

\section{I volti della follia}

Sebbene la psichiatria forense e la criminologia abbiano ormai da tempo smentito tale convinzione, pare, dunque, riaffacciarsi il secolare pregiudizio in base al quale l'infermo psichico è pericoloso in quanto tale. Al di là delle questioni contingenti che, come si è rilevato, possono aver influenzato il legislatore nella formulazione di tale normativa, una domanda che risulta interessante porsi è per quale motivo l'associazione fra malattia mentale e violenza, di lombrosiana derivazione, pare così tenacemente radicata.

Si può considerare la patologia psichica come solo uno dei volti, quello più recente e medicalizzato, della follia, concetto ben più ampio e che implica ambigue e mai risolte commistioni. Di fronte al reo malato di mente, infatti, non solo il mondo giuridico, ma prima ancora la percezione comune, tende a vedere giustapposte cura e repressione, terapia e controllo sociale, esigenza di difesa e necessità di riconoscergli dignità umana. La nozione di pericolosità sociale psichiatrica raccoglie l'eredità di questa "confusione antropologica" che ha radici lontane e che il filosofo francese Michel Foucault ha cercato, tra i primi, di cogliere e di rappresentare nella Storia della follia nell'età classica: un'opera, pubblicata nel 1961, che spiazza, divide, affascina, ma sicuramente non lascia, ancora oggi, indifferenti (Ugolini, 2013, p. 84).

Le argomentazioni di Foucault nella Storia della follia sono espressione di un metodo che egli definisce "archeologico": non si tratta, però, di portare alla luce avvenimenti omessi o trascurati, ma di riconsiderare i fatti storici nella loro particolarità unica e irripetibile di eventi, evitando di volerli forzatamente disporre secondo un ordine e una regolarità, tanto rassicuranti quanto apparenti. L'"archeologo" non si occupa, quindi, di dispiegare una storia delle idee o dei saperi, ma studia piuttosto i presupposti per la comparsa e i principi di costruzione dei discorsi; ben conscio, inoltre, della difficoltà di poter indicare un chiaro ed evidente susseguirsi di cause per porre i diversi fatti in correlazione. Ciò che abbiamo di fronte, secondo Foucault, sono, invece, un insieme di eventi di cui dobbiamo rilevare, caso per caso, le condizioni, i confini, le relazioni e, soprattutto, le "fratture" (Foucault, 2009). Nella Storia della follia non viene, dunque, esposto il risultato di una ricerca cronologicamente lineare, ma qualcosa di più complesso e, al contempo, meno individuabile come sistematico: la ricostruzione dei cambiamenti nella consapevolezza culturale e nell'atteggiamento che la società adotta nei confronti dei folli, tra XVII e XVIII secolo. Foucault, in altri termini, segue, porta allo scoperto e delinea la comparsa della basilare esperienza di ciò che chiamerà sragione (Foucault, 1998).

Ciò che si verifica, in quella che in Francia viene definita età classica, è una giustapposizione tra l'esperienza della follia come malattia e l'esperienza in cui, invece, la follia appartiene alla punizione e alla correzione. Si tratta di due forme di alienazione diverse che fino al Settecento si sovrappongono senza arrivare a congiungersi: l'una intesa come limitazione dei poteri e della libertà del soggetto ritenuto irresponsabile; l'altra che, al contrario, designa il folle come l'Altro, come lo Straniero, come l'Escluso. A parere di Foucault, quando, successivamente, si deciderà di fare dell'internamento un atto terapeutico che si propone di guarire un malato, incapace e folle, verranno riuniti di colpo, in una unità confusa, i molteplici volti della follia. Nel Seicento, il trattamento inquisitoriale del folle viene ancora applicato, ma questo è il secolo dove ha inizio quello che Foucault ha, appunto, definito il grande internamento (Ugolini, 2013, p. 84). In questo periodo, tutta una variopinta popolazione si trova rigettata in uno spazio sociale che, benché nato dalla grande inquietudine di fronte alla miseria, non è affatto lo spazio della povertà e non è ancora quello della malattia. Le misure che portano all'internamento riflettono un insieme di operazioni che, in sordina, elaborano un dominio di esperienza nel quale la follia si riconoscerà prima di prenderne possesso: si tratta di operazioni che spostano i confini della moralità, stabiliscono nuovi veti, mitigano vecchi scandali. La sragione prende l'aspetto di tipi, di personaggi quali il mago, la strega, l'alchimista, il libertino, l'omosessuale, il suicida, e anche gli "insensati". Questi ultimi occupano un posto particolare nel mondo dell'internamento, poiché si tratta degli "alienati": siamo qui nel campo della follia propriamente detta. Fra questi tipi, mescolati nell'internamento, si intrecciano parentele e comunicazioni, si delimita un campo quasi omogeneo, si crea un reticolato sotterraneo che delinea, 
in qualche modo, i segreti fondamenti della nostra esperienza moderna della follia (Foucault, 1998).

Le formule dell'internamento non predicono quelle che saranno le nostre malattie, ma indicano forme di follia e, soprattutto, pongono in evidenza una percezione morale della follia. Nell'età classica, secondo Foucault, non c'è nessuna disgiunzione tra follia e colpa, tra alienazione e malvagità: «non sono né considerati come criminali, né trattati come malati o come personaggi sacri. Tuttavia sono un po' simili ai criminali, ai malati, ai personaggi sacri. Eppure il loro statuto è diverso. [...] Vedete che, di conseguenza, è possibile designare tutto quello che chiamerei la parentela sociologica del folle. Questo personaggio folle che si trova in tutte le società, è prossimo al malato, al deviante sessuale e familiare, è prossimo al personaggio sacro, e tuttavia non si identifica esattamente con nessuna di queste categorie» (Foucault, 2005, pp. 294-297). L'elemento che attraversa tale parentela sociologica è il disordine, sia esso fisico, sociale, morale, religioso, sessuale, di cui il folle sembra portatore o con cui, comunque, sembra apparentato. Disordine che racchiude in sé una violenza che attiene alla dimensione del Caos, a un nucleo magmatico di irrequieta imprevedibilità che non sappiamo bene, nel suo agitarsi continuo, più o meno sotterraneo, quale forma e direzione prenderà (Ugolini, 2013, p. 85).

Attualmente, gli studi che riguardano il rapporto tra violenza e malattia psichica sembrerebbero ridimensionare l'idea per cui un malato psichico è necessariamente violento, anche se con alcuni importanti distinguo. Se è vero, infatti, che la percentuale di omicidi commessi non è più elevata tra i malati di mente rispetto alla popolazione generale (Dodaro, 2011, p. 178), è vero anche che quando essi uccidono o commettono violenze le loro principali vittime sono i familiari: «ben più spesso dei sani di mente gli omicidi alienati uccidono familiari o parenti: i quali costituiscono oltre i $2 / 3$ delle loro vittime; all'opposto, è ben più raro fra gli alienati che fra i soggetti normali l'omicidio di persone sconosciute» (Canuto \& Tovo, p. 330). Riguardo, in particolare, agli schizofrenici, l'American Psychiatric Association ha sottolineato che la maggior parte di loro non è più pericolosa per gli altri rispetto a un qualunque soggetto dotato di normale salute mentale; tuttavia, occorrerebbe anche aggiungere che molti studi hanno riportato che i soggetti affetti da schizofrenia hanno un'incidenza più elevata di comportamento aggressivo e violento. Anche patologie più diffuse e non propriamente classificate come psicotiche possono, inoltre, determinare manifestazioni di grande aggressività: i soggetti affetti da disturbo bipolare, ad es., durante gli episodi maniacali, possono manifestare «violenza verso i bambini, verso il coniuge o altri comportamenti violenti» (American Psychiatric Association, 2007, p. 333).

E' la "mania", a differenza della "malinconia", a identificare, sin da Ippocrate, l'aspetto aggressivo della follia ritenuta nell'antichità la conseguenza di un eccesso di bile gialla nel cervello (Giurovich, 2004, pp. 161-163); lo stesso eccesso di tale umore avrebbe determinato, non a caso, il cosiddetto temperamento collerico predisposto, più degli altri, ad incorrere nella mania. Ancora nell'Ottocento, la mania esteriorizza gli aspetti più bellicosi della follia, poiché viene definita come «alienazione mentale che porta per carattere principale la manifestazione dell' incoerenza dei pensieri e delle volizioni, con impeto, con violenza e con furore» (Ferrarese, 1830, p. 2). Proprio il furore è, per Foucault, l'aspetto della follia che accompagna e simbolizza in modo omogeneo il grande internamento avvenuto in età classica. II furore fa riferimento «a tutte le forme di violenza che sfuggono alla definizione rigorosa del delitto e alla sua catalogazione giuridica; esso mira [...] a tutto il dominio oscuro di una rabbia minacciosa che appare al di qua di una possibile condanna». Dire di qualcuno che era furioso significava poterlo internare, senza dover precisare se malato o criminale (Foucault, 1998, p. 114).

E' questo furore sempre percepito come latente, anche nelle forme più silenziose e innocue di follia, che rende il folle perennemente qualcosa di altro e di diverso rispetto a un semplice malato da curare. Nel caso particolare e odierno della follia come malattia mentale, infatti, non possiamo guarire il paziente: possiamo controllarlo, contenerlo, sedarlo, migliorare anche indefinitamente la sua qualità di vita, possiamo stabilire correlazioni mediche e comportamentali che hanno un alto grado di probabilità, ma non possiamo stabilire con certezza se e quando la crisi o lo scompenso si presenteranno, con quali effetti, con quale intensità. E, soprattutto, non possiamo prevedere, se non con approssimazioni riguardanti il probabile livello di rischio, se la violenza del malato irromperà verso se stesso, verso i familiari, verso la comunità. Alla malattia mentale continua, così, in modo obliquo e silenzioso, a venire associata una violenza inaspettata, immotivata, casuale 
(Ugolini, 2013, p. 85).

Pur non identificandosi propriamente con nessuna delle tipologie di devianza, il folle è vicino a tutte e, in qualche misura, a tutte apparentato. A differenza di esse, però, il disordine che segna il folle si esprime, in atto o in potenza, come violenza caotica. Ma c'è anche altro. Spesso la follia pare assumere contorni più inquietanti. Secondo quanto sostiene Foucault, «il soggetto può essere un po' più folle o un po' più criminale, la più estrema follia sarà fino in fondo inquinata di malvagità. [...] Nel mondo dell'internamento la follia non spiega e non scusa niente: essa entra in complicità con il male per moltiplicarlo, per renderlo più insistente e pericoloso e prestargli nuovi volti» (Foucault, 1998, pp. 139-140). In tale prospettiva, la follia deriva da un cedimento della volontà e, in quanto tale, è considerata come un errore etico. La follia comunica con il Male attraverso la scelta individuale e l'intenzione malvagia. Essa entra, dunque, in complicità con il Male per moltiplicarlo e prestargli nuovi volti (Foucault, 1998, pp. 137-142).

Occorre sottolineare che tale correlazione privilegiata tra male e follia, che Foucault evidenzia trattando del grande internamento, ha radici antiche. Nel Medioevo il folle apparteneva al sacro: una manifestazione di Dio o del diavolo. Egli esprimeva l'antagonismo tra bene e male e rappresentava un'occasione per vincere il demonio. A partire dall'istituzione dell'Inquisizione, il pazzo viene accomunato all'eretico: entrambe saranno considerate maschere del male da punire con la tortura e il rogo. Nel Malleus maleficarum, il manuale di procedura inquisitoriale che giustificò la messa al rogo di un numero straordinario di donne, le descrizioni del demonio e delle streghe delineano, secondo Andreoli (1991, p. 9), quadri psichiatrici. Se non tutte le persone accusate di stregoneria e di commerci demoniaci erano considerate inferme di mente, è vero, tuttavia, il contrario: quasi tutti gli infermi di mente erano considerati streghe o legati, comunque, a patti diabolici (Stok, 2000, pp. 165-167). Di tale inquietante complicità, si è, ovviamente, occupata in modo circostanziato la prospettiva teologica. Non a caso, dobbiamo rilevare come l'alleanza tra follia e male trovi una sua significativa testimonianza in quelli che, secondo la Chiesa Cattolica, sono i criteri diagnostici per individuare una possessione diabolica. La certezza di una possessione dipende, infatti, dal verificarsi congiunto di alcune condizioni: una di queste è proprio la presenza, nel soggetto, di una fenomenologia di tipo psichiatrico che, il più delle volte, viene a coincidere con il disturbo dissociativo d'identità (Ugolini, 2013, p. 69). Ritroviamo, quindi, anche lo psichiatra, accanto al sacerdote esorcista, quando occorre discernere se un soggetto è un "semplice" malato mentale o qualcuno che ha subito, invece, quello che è considerato il grado estremo e straordinario dell'azione demoniaca (Balducci, 1994).

Nella mitologia greca, Pan è una delle poche divinità a cui è direttamente riconducibile il disturbo mentale; nella visione cristiana, diventerà una delle figure associate al diavolo (Hillman, 1977); Pan sarà, poi, interpretato, dai satanisti moderni, come uno dei 77 demoni da invocare durante le cerimonie (La Vey, 1969). L'occultismo europeo accoglie la follia, in una sorta di tenebrosa alleanza, in particolare nelle cerimonie di magia nera, laddove è richiesto al mago non il distacco ascetico ma, al contrario, la sfrenatezza delle passioni. Aleister Crowley, il più discusso e famoso occultista del Novecento, sostiene come sia necessario che l'Ego conscio del mago sia distrutto per poter essere assorbito in quello del dio invocato: in altri termini, occorre trascendere la propria coscienza individuale, estinguendo il proprio lo usuale per raggiungere fini rituali distruttivi. Ciò comporta uno stato di esaltazione e di frenesia dirompente, una eccitazione ingovernabile, qualcosa di analogo alle ebbrezze della pura sragionevolezza. Secondo Crowley (1976), infatti, la mente deve essere spezzata in una forma di follia prima che, durante il rito magico, possa venire trascesa.

\section{Conclusioni}

L'ambiguo sovrapporsi tra l'esperienza della follia come malattia e l'esperienza in cui, invece, la follia appartiene alla punizione e alla correzione è un'eredità con cui tuttora facciamo i conti. Ancora oggi abbiamo a che fare, nella percezione sociale e nella esperienza giuridica, con tale ambivalenza: di fronte al malato di mente tendiamo a vedere giustapposte cura e repressione, terapia e controllo sociale, esigenza di difesa dalle sue imprevedibili aggressività e necessità di riconoscergli dignità umana e diritti. La nozione di pericolosità sociale psichiatrica raccoglie l'eredità 
di questa "confusione antropologica".

Per quanto il diritto possa avere la volontà e gli strumenti per correggere, perfezionare o anche abolire l'istituto della pericolosità, probabilmente rimarrà tenace la tendenza a percepire sempre insieme i diversi volti della follia, senza mai riuscire a considerarli del tutto separatamente. Si potrà mai considerare il folle, tanto più se colpevole di reato, soltanto come un malato da curare? Incapace, una volta contenuto farmacologicamente, di qualsiasi manifestazione di "furore"? Non esiste psicofarmaco, per quanto sicuro e sperimentato, che possa allontanare dalle nostre coscienze la paura del folle e dei suoi gesti imprevedibili, poiché egli appartiene a una alterità ontologicamente lontana, che non si esaurisce in nessuna conoscenza medica o giuridica.

\section{References}

American Psychiatric Association (2007). DSM-IV-TR. Manuale diagnostico e statistico dei disturbi mentali. Milano: Masson.

Andreoli, V. (1991). Un secolo di follia. Milano: Rizzoli.

Balducci, C. (1994). II diavolo. Milano: Mondadori.

Bandini, T., \& Rocca, G. (2010). Fondamenti di psicopatologia forense. Milano: Giuffrè.

Bianchetti, R. (2014). Sollevata questione di legittimità costituzionale in merito ai nuovi criteri di accertamento della pericolosità sociale del seminfermo di mente. http://www.penalecontemporaneo.it/ (14 novembre 2014).

Canuto, G., \& Tovo, S. (1996). Medicina legale e delle assicurazioni. Padova: Piccin.

Crowley, A. (1976). Magick. Roma: Astrolabio.

Dodaro, G. (2011). La posizione di garanzia degli operatori psichiatrici. Giurisprudenza e clinica a confronto. Milano: Franco Angeli.

Ferrarese, L. (1830). Delle malattie della mente ovvero delle diverse specie di follie, Napoli.

Foucault, M. (1998). Storia della follia nell'età classica, Milano: BUR.

Foucault, M. (2005). Follia e psichiatria. Detti e scritti (1957-1984). Milano: Cortina.

Foucault, M. (2009). L'archeologia del sapere. Milano: BUR.

Giurovich, S. (2004). Problemi e metodi di scienza ippocratica: testi e commenti. Bologna: Pendragon.

Hillman, J. (1977). Saggio su Pan. Milano: Adelphi.

La Vey, A., S. (1969). The satanic bible. Secaucus (N.J.): University Books.

Massaro, A. (2015). Sorvegliare, curare e non punire: l'eterna dialettica tra "cura" e "custodia" nel passaggio dagli ospedali psichiatrici alle residenze per l'esecuzione delle misure di sicurezza. Rivista Italiana di Medicina Legale, 4, 1358-1360.

Pelissero, M. (2014). Ospedali psichiatrici giudiziari in proroga e prove maldestre di riforma della disciplina delle misure di sicurezza. Diritto penale e processo, 8, 917-930.

Pugiotto, A. (2015). La giurisprudenza difensiva in materia di OPG a giudizio della Corte Costituzionale. Rivista dell'Associazione Italiana dei Costituzionalisti, 4, 3.

Stok, F. (2000). La lunga storia del manicomio. In L. Attenasio (Ed.), Fuori norma. La diversità come valore e sapere (pp. 165-167). Roma: Armando.

Ugolini B. (2013), Diritto e psichiatria dopo la legge 180, tesi di dottorato in http://ecum.unicam.it

\section{Websites}

www.giurcost.org

www.stopopg.it 